\title{
Analysis of empirical parametrization and microscopical studies of deuteron- induced reactions
}

\author{
Marilena Avrigeanu*, Cristian Costache, and Vlad Avrigeanu \\ ${ }^{1}$ Horia Hulubei National Institute for Physics and Nuclear Engineering, P.O. Box MG-6, 077125 Bucharest-Magurele, Romania
}

\begin{abstract}
A review of deuteron-induced reaction analyses is carried out paying due consideration to reaction cross-section parametrization as well as theoretical models associated to the deuteron interaction process. The key role of direct interactions, i.e., breakup, stripping and pick-up processes is stressed out by the comparison of data with theoretical and evaluation predictions, including the latest TENDL-2017 library.
\end{abstract}

\section{Introduction}

On-going strategic research programs (ITER, IFMIF, SPIRAL2-NFS) [1] and medical investigations using accelerated deuterons triggered even a decade ago an update of the theoretical analysis of deuteron activation cross sections within the FENDL-library project [2]. This update was motivated essentially by the specific noncompound processes that should be considered in the case of the incident deuterons, making them substantially different from other incident particles. Thus, the deuteron breakup (BU) is particularly quite important due to the large variety of reactions initiated by the breakup nucleons along the whole incident energy range. Otherwise, the deuteron interaction with low and medium mass target nuclei and incident energies below and around the Coulomb barrier proceeds largely through stripping, $(d, p)$ and $(d, n)$, and pick-up, $(d, t)$ and $(d, \alpha)$, direct reaction (DR) mechanisms, while pre-equilibrium emission (PE) and evaporation from fully equilibrated compound nucleus $(\mathrm{CN})$ become important at higher energies $[3,4]$.

More recently, full parametrization of the deuteron monitor reactions and therapeutic radionuclidesproduction cross sections have been recommended within Special Issues on Nuclear Reaction Data by Hermanne et al. [5], and Engle et al. [6]. Thus, genuine Padé fit of the available data has been involved at variance, however, with the FENDL [2] concern of deuteron-induced reaction improved theoretical analysis. Actually, Engle et al. [6] motivated the choice of Pade fit there, despite so low predictive power and apart from nuclear modeling advance, by the deficiency in the theoretical description of the deuteron breakup. Therefore it seems appropriate a comparative analysis of empirical parametrization and microscopic studies within the experimental data and theoretical predictions leading to a final evaluation of deuteron data.

\section{Additive reaction cross-section parametrization and model analysis}

A particular case in this respect could be the interconnection of two valuable complementary experimental data sets of ${ }^{231} \mathrm{~Pa}(d, 3 n)^{230} \mathrm{U}$ and ${ }^{231} \mathrm{~Pa}(p, 2 n)^{230} \mathrm{U}[8]$ excitation functions, measured between [11.2 - 19.9 MeV] [7], and [10.6 - 23.8 MeV], respectively, which have also been analyzed separately by Padé fit due the deficiency in the theoretical deuteron-BU description [6]. On the other hand, we pointed out earlier $[9,10]$ the dominant role of the breakup mechanism in the interaction process of deuterons with Actinides targets at incident energies around Coulomb barrier. The proper handling of both breakup components, the elastic breakup (EB), in which the target nucleus stays in its ground state, and the inelastic breakup (BF), where one of the breakup nucleons interacts nonelastically with the target nucleus, leads to the description of the ${ }^{231} \mathrm{~Pa}(d, 3 n)^{230} \mathrm{U}$ excitation function, as shown by the solid curve in Fig. 1 [9].

On the whole, the leakage of the initial deuteron flux toward the breakup process reduces the total reaction cross section $\sigma_{R}$ that should be shared among different outgoing channels by a reduction factor $\left(1-\sigma_{B U} / \sigma_{R}\right)[3,4,9,10]$, where $\sigma_{B U}$ is the total breakup cross section. This effect is shown in Fig. 1 for the ${ }^{231} \mathrm{~Pa}(d, 3 n){ }^{230} \mathrm{U}$ reaction comparing $\mathrm{PE}$ and $\mathrm{CN}$ mechanisms contribution to $(d, 3 n)$ reaction without (dash-dot-dotted curve) and with (dot-dashed curve) inclusion the correction for the incident flux leakage through the breakup [9]. On the other hand, a subsequent interaction between one of the deuteron constituents and the same target nucleus may lead to enhancement of various reaction channels. Here, the particular value of the ${ }^{231} \mathrm{~Pa}(p, 2 n)^{230} \mathrm{U}$ reaction cross-section measurements [8] comes from the superposition of its incident-energy range and the breakup proton energies corresponding to the energy range of the reaction ${ }^{231} \mathrm{~Pa}(d, 3 n)^{230} \mathrm{U}$. In this particular case, the interaction with the target nucleus of a breakup proton enhances through $(p, 2 n)$ reaction the

*e-mail: marilena.avrigeanu@nipne.ro 


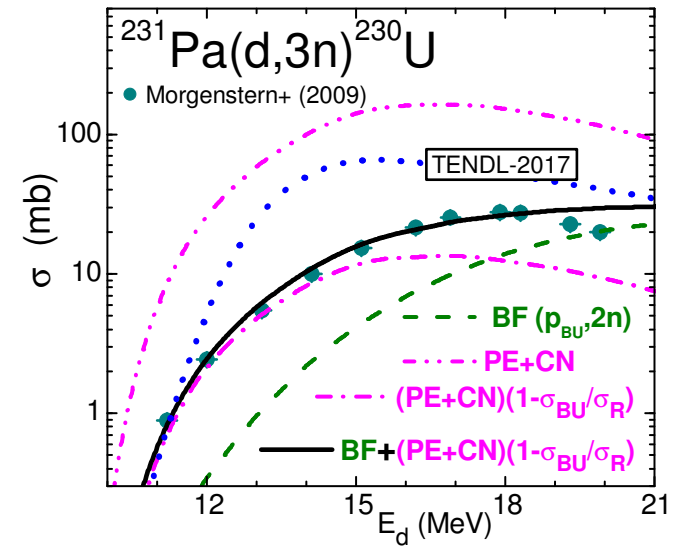

Figure 1. Comparison of the measured [7], latest TENDL2017 [11] evaluation (dotted curve) and model calculation (solid curve) including the BF (dashed curve), and $\mathrm{PE}+\mathrm{CN}$ contribution to ${ }^{231} \mathrm{~Pa}(d, 3 n)^{230} \mathrm{U}$ reaction cross sections calculated without (dash-dot-dotted) and with (dot-dashed) inclusion of the BU effect on $\sigma_{R}[9]$.

$(d, 3 n)$ residual channel population (dashed curve in Fig. 1 [9]). This is why the simultaneous analysis of $(d, 3 n)$ [7] and $(p, 2 n)$ excitation functions is so useful for the study of the inelastic breakup and complementary reaction mechanisms considered for the deuteron interactions with nuclei.

Overall, the enhancing effect of the breakup mechanism is important mainly for describing the excitation functions for second and third chance emitted-particle channels [3, 4, 9] (e.g., see dashed curves in Figs. 1, 2, and 4).

Concerning the deuteron monitor reactions described with Padé fit by Hermanne et al. [5], almost all of them have already been analyzed in the frame of BU, DR, PE and $\mathrm{CN}$ reaction mechanisms models $[3,4]$. These analyzes covered the whole experimental systematics of the deuteron induced reactions on the natural element target and its isotopes, making possible a reliable understanding of the interaction process. Confidence in the reaction cross-section predictions at energies where the measurements still not exist, aimed by the FENDL project, has thus been provided.

\section{Deuteron breakup}

Our description of the deuteron breakup mechanism is based on the parametrization [12] of both the total breakup proton emission $\sigma_{B U}^{p}$ and $\sigma_{E B}$ cross sections, assuming equal inelastic-breakup cross sections for the breakup neutron and proton. It has been obtained through analysis of the experimental systematics of deuteron-induced reactions on target nuclei from ${ }^{27} \mathrm{Al}$ to ${ }^{232} \mathrm{Th}$ and incident energies up to $80 \mathrm{MeV}$ [13].

The comparison of the measured $\sigma_{B U}^{p}$ at 15, 25.5, 56, 70 , and $80 \mathrm{MeV}$ deuteron energies and for target nuclei from ${ }^{12} \mathrm{C}$ to ${ }^{232} \mathrm{Th}$ [13], with (i) the above parametrization as well as the microscopic cross sections obtained in the frame of (ii) the CDCC extension of the eikonal reaction theory (ERT), using microscopic optical potentials by Neoh et al. [14], and (iii) distorted wave Born approximation (DWBA) method with post form interaction and zero-range approach by Carlson et al. [15], is shown in Fig. 3 (a-d). Since the absolute cross sections may depend on the model ingredients of reaction mechanisms involved within the experimental data analysis, a similar comparative analysis concerns at the same time in Fig. 3 (e-h) the corresponding total breakup proton-emission fractions $f_{B U}^{p}$ $=\sigma_{B U}^{p} / \sigma_{R}$. Moreover, the $f_{B U}^{p}$ values may illustrate the importance of the breakup process among the other reaction mechanisms related to the deuteron interactions. The same scale has been used for $\sigma_{B U}^{p}$ as well as $f_{B U}^{p}$ values at all incident energies of the available data.

There are several features which are pointed out by this comparative analysis. First, the increase of $\sigma_{B U}^{p}$ with the target-nucleus mass is well described by the empirical parametrization for all deuteron energies from 15 to $80 \mathrm{MeV}$. There is a similar trend of the microscopic results for medium-mass nuclei with $40<A<120$, while it is apparent an overestimation of the measured data for light nuclei $(A<40)$ at both 25 and $56 \mathrm{MeV}$ incident energies, as well as an underestimation for heavier ones $(A>120)$. Second, the importance of the BU mechanism, shown by $f_{B U}^{p}$, is increasing with the target-nucleus mass, from ${ }^{27} \mathrm{Al}$ up to ${ }^{232} \mathrm{Th}$, at the lower incident energies of 15 and 25.5 $\mathrm{MeV}$. This increase is less significant at the energy of 56 $\mathrm{MeV}$, and even reversed at 70-80 MeV. Actually it seems that the fraction $f_{B U}^{p}$ has reached its maximum at $56 \mathrm{MeV}$, for the target nuclei with $A>120$, while for $40<A<120$ this maximum moves at energies over $56 \mathrm{MeV}$ but lower than 70-80 MeV. Moreover, the $f_{B U}^{p}$ values are still increasing with the incident energy even at $80 \mathrm{MeV}$ for the deuteron interaction with light target nuclei $(A<40)$. These energy dependencies of the measured $f_{B U}^{p}$ are satisfactorily described by the empirical parametrization. The microscopic results at $25 \mathrm{MeV}$ [15] show almost constant $f_{B U}^{p}$ for the whole A interval analyzed, while at $56 \mathrm{MeV}[14,15]$ show a steep decrease for target nuclei from $A=12$ up to $A \sim 120$, apart from the data, while for $A>120$ their underestimated values are obvious.

\section{Direct reactions}

The interactions of deuterons with medium-mass target nuclei at energies around the Coulomb barrier proceed largely through the DR mechanism [3, 4] which is therefore quite important for the cross sections related to the first-chance emitted particle within $(d, p),(d, n),(d, t)$, and $(d, \alpha)$ residual channels. Thus, the assessment of the total transfer reaction cross section is mandatory, in spite of very poor attention or even not accounted so far in deuteron activation analysis. The calculation of DR cross sections has been performed using the DWBA formalism within the FRESCO code [17]. The post/prior form distorted-wave transition amplitudes for the stripping and pick-up reactions, respectively, and the finite-range interaction have been considered. The spectroscopic factors have been obtained from the DWBA analysis of the experimental angular distributions of the stripped/picked parti- 

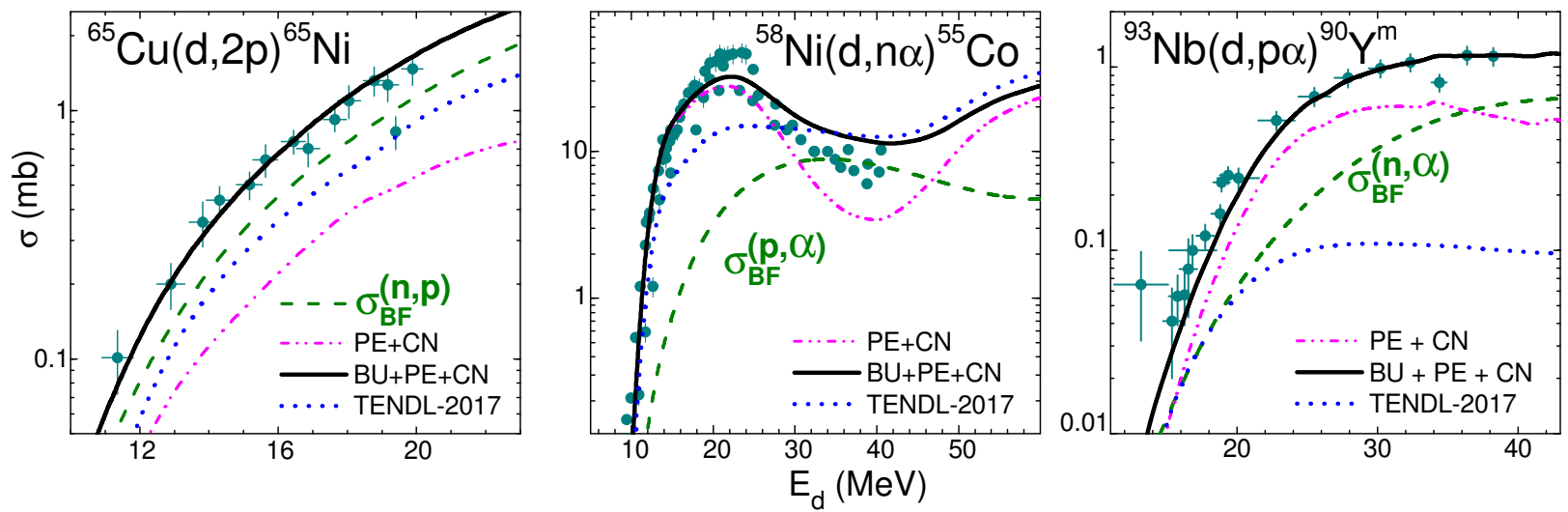

Figure 2. Comparison of measured deuteron activation cross sections [16], the TENDL-2017 evaluations (dotted curves) [11], and the model calculations (solid curves) [3,4] taking into account the BF (dashed curves) and PE+CN contributions (dash-dot-dotted curves).

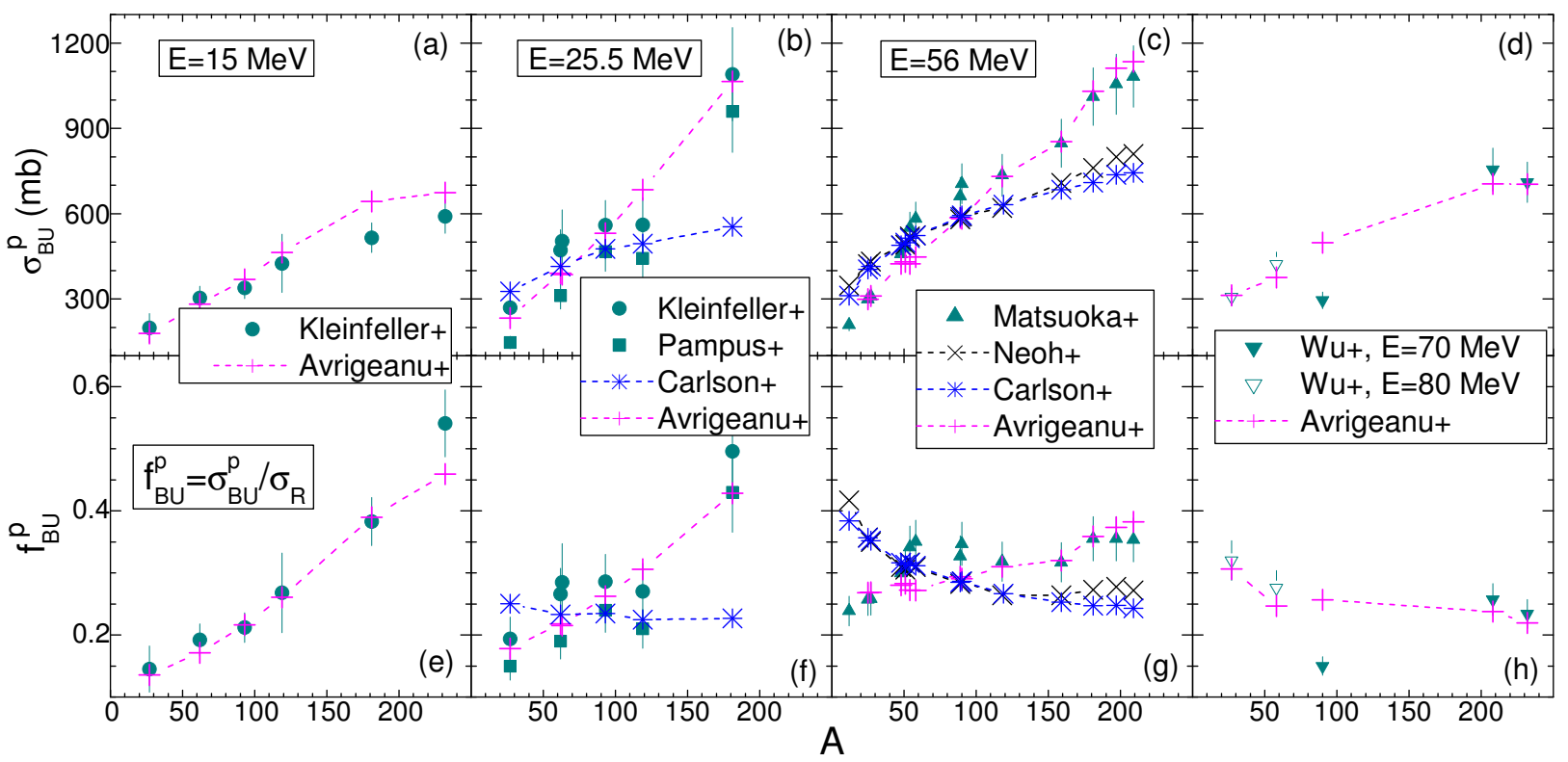

Figure 3. Comparison of the mass dependence of measured (solid circle, $\mathbf{E}, \mathbf{\Lambda}, \mathbf{\nabla}, \nabla)$ [13] total BU proton-emission cross sections (top) and fractions (bottom) with the predictions of the microscopic eikonal model [14] ( $\times$ ), DWBA formalism [15] (*), and of the empirical parametrization $(+)$ [12], connected by dashed lines for eye guiding, for target nuclei from ${ }^{12} \mathrm{C}$ up to ${ }^{209} \mathrm{Bi}$, at the incident energies of $15,25.5,56,70$, and $80 \mathrm{MeV}$.
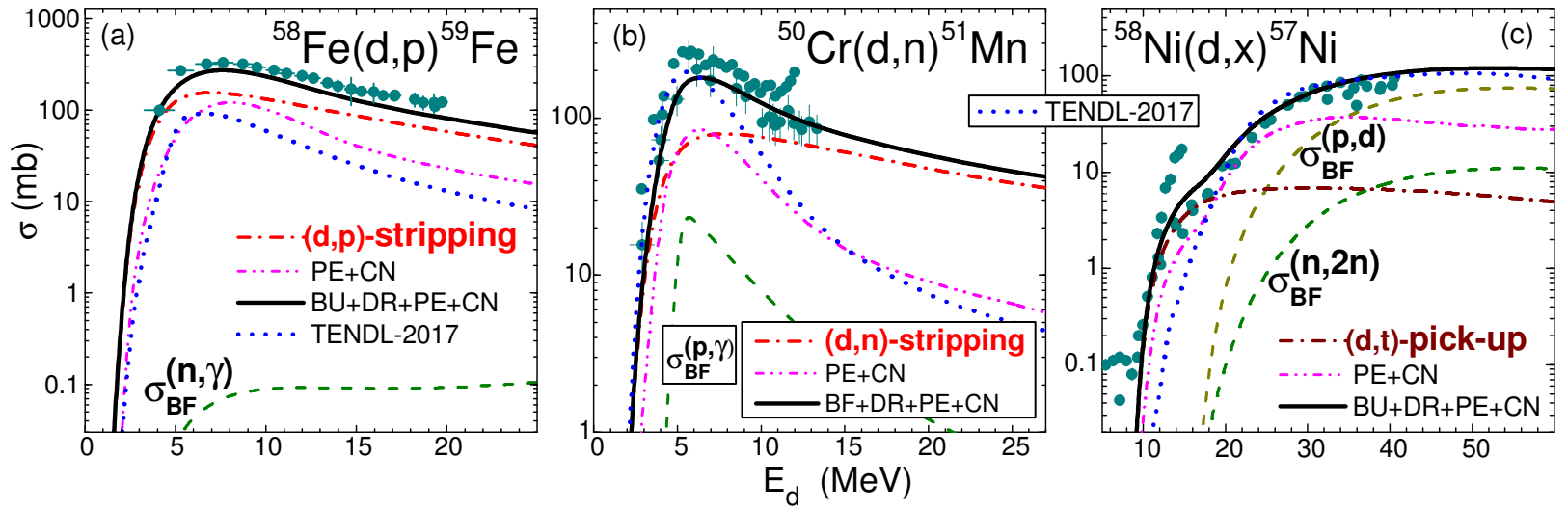

Figure 4. Same as Fig. 2 but including the DR (dot-dashed curves) contributions, important for the first chance emitted particle [4]. 
cles as presented in the detailed descriptions of the input given elsewhere $[3,4]$.

The significant effects of the stripping and pick-up reactions for the deuteron interaction with ${ }^{58} \mathrm{Fe},{ }^{54} \mathrm{Fe}$, and ${ }^{58} \mathrm{Ni}$ target nuclei, respectively, have been reassessed in Fig. 4. It is thus proved that the direct reactions are quite important for the first-chance particle emission, the stripping mechanism being the dominant one for the $(d, p)$ reactions [3, 4] [Fig. 4(a)]. A particular note should also concern the pick-up essential contribution to the total $(d, t)$ activation cross section at the energies between its threshold and those for the $(d, n d)$ and $(d, 2 n p)$ reactions that lead to the same residual nucleus [Fig. 4(c)].

\section{Statistical particle emission}

The statistical $\mathrm{PE}+\mathrm{CN}$ reaction mechanisms, which complete the deuteron interaction analysis along an enlarged nuclear-interaction time scale, become important with the increase of the incident energy above the Coulomb barrier. The corresponding reaction cross sections have been calculated using the STAPRE-H code [18] and various versions of TALYS code [19], taking into account the overall reduction of the deuteron flux due its absorption in $\mathrm{BU}$ and DR processes [3, 4]. Another particular point of these calculations is the use of the same common model parameters to account for different reaction mechanisms.

The appropriate description of nuclear mechanisms involved within deuteron-induced reactions is validated by an overall agreement of the calculated and measured cross sections as shown, e.g., in Figs. 1, 2, 4 [3, 4, 9]. The mark $\mathrm{BU}$, rather than $\mathrm{BF}$, for the sum of various contributions to an activation cross section in these figures underlines the consideration of both breakup effects, i.e., the overall decrease of $\sigma_{R}$ as well as the BF enhancement. On the other hand, the apparent discrepancies between the experimental data and corresponding TENDL-2017 [11] latest evaluation shown in the above figures stress out the effects of disregarding the direct processes (breakup, stripping, pick-up) within TENDL evaluation.

\section{Conclusions}

The present work has concerned actually a review of deuteron-interaction analysis stressing out the key role of direct interactions, namely the breakup process and direct reactions. The overall agreement between the measured data and model calculations sustains the description of nuclear mechanisms taken into account for the deuteronnucleus interaction, emphasizing that the neglected direct interactions should be considered responsible for the discrepancies shown by the current evaluation predictions. The consistent theoretical frame of the deuteron interactions supported by advanced codes associated to the nuclear reactions mechanisms provides predictability in addition to the use of various-order genuine Pade approximations $[5,6]$
The recently increased interest on the theoretical analysis of the breakup components [14, 15] may lead eventually to the refinement of the deuteron breakup empirical parametrization and increased accuracy of the deuteron activation cross section calculations.

This work was partly supported by Autoritatea Nationala pentru Cercetare Stiintifica (Project PN-19060102) and Euratom research and training program 2014-2018 and 2019-2020 under Grant Agreement No. 633053. The views and opinions expressed herein do not necessarily reflect those of the European Commission.

\section{References}

[1] www.iter.org, www.ifmif.org, www.ganil-spiral2.eu

[2] Fusion Evaluated Nuclear Data Library (FENDL) 3.0, https://www-nds.iaea.org/fendl3/

[3] P. Bém et al., Phys. Rev. C 79, 044610 (2009); E. Šimečková et al., ibid. 84, 014605 (2011), ibid. 98, 034606 (2018).

[4] M. Avrigeanu et al., Phys. Rev. C 88, 014612 (2013); ibid. 89, 044613 (2014); ibid. 92, 021601(R) (2015); ibid. 94, 014606 (2016); M. Avrigeanu and V. Avrigeanu, Nucl. Data Sheets 118, 301 (2014).

[5] A. Hermanne et al., Nucl. Data Sheets 148,338(2018).

[6] J.W. Engle et al., Nucl. Data Sheets 155, 56 (2019).

[7] A. Morgenstern et al.,Phys. Rev. C 80, 054612 (2009).

[8] A. Morgenstern et al., Anal. Chem. 80, 8763 (2008).

[9] M. Avrigeanu et al., Phys. Rev. C 85, 034603 (2012).

[10] M. Avrigeanu and V. Avrigeanu, J. Phys: Conf. Ser. 724, 012003 (2016); ibid. 1023, 012009 (2018); EPJ Web of Conferences 146, 012020 (2017).

[11] A.J. Koning and D. Rochman, TENDL-2017: TALYS-based evaluated nuclear data library, https://tendl.web.psi.ch/tendl_2017/tendl2017.html

[12] M. Avrigeanu and V. Avrigeanu, Phys. Rev. C 95, 024607 (2017); M. Avrigeanu et al., Fusion Eng. Design 84, 418 (2009).

[13] J. Pampus et al., Nucl. Phys. A 311, 141 (1978); J.R. Wu et al., Phys. Rev. C 19370 (1979); N. Matsuoka et al., Nucl. Phys. A 345, 1 (1980); J. Kleinfeller et al., Nucl. Phys. A 370, 205 (1981).

[14] Y. S. Neoh et al., Phys. Rev. C 94, 044619 (2016).

[15] B. V. Carlson et al., Few-Body Syst. 57, 307 (2016).

[16] Experimental Nuclear Reaction Data (EXFOR), https://www-nds.iaea.org/exfor/

[17] I.J. Thompson, Comput. Phys. Rep. 7, 167 (1988), v. FRES 2.9 (2011).

[18] M. Avrigeanu and V. Avrigeanu 1995 IPNE Report NP-86-1995, Bucharest, and Refs. therein; http://www.oecdnea.org/tools/abstract/detail/iaea0971/; Comp. Phys. Comm. 112, 191 (1998); A.Harangozo et al., Phys. Rev. C 58, 295 (1998).

[19] A.J. Koning, S. Hilaire, and S. Goriely, v. TALYS1.9, 2017, http://www.talys.eu 